\title{
Connecting Correction Methods for Linkage Error in Capture-Recapture
}

\author{
Peter-Paul de Wolf ${ }^{1}$, Jan van der Laan ${ }^{1}$, and Daan Zult ${ }^{1}$
}

\begin{abstract}
A commonly known problem in population size estimation using registers, is that registers do not necessarily cover the whole population. This may be because they intend to cover part of the population (e.g., students), due to administrative delay or because part of the target population is not registered by default (e.g., illegal persons). One of the methods to estimate the population size in the presence of undercount is the capture-recapture method that combines the information of two or more samples. In the context of census estimation registers are used instead of samples. However, the method assumes that perfect linkage between the registers can be achieved. It is known that this assumption is often violated.

In the setting of evaluating the population coverage of a census using a post-enumeration survey, a correction for linkage error was proposed. That correction was later generalized by relaxing some of the newly introduced conditions. However, the new correction method still implicitly assumed that the two registers are of equal size. We introduce a further generalization that includes both previously mentioned correction methods and at the same time deals with registers of different sizes. Specific parameter settings will correspond to the different correction methods. We show that the parameters of each method can be chosen such that the resulting estimates all equal the traditional Petersen estimate (1896) that would theoretically be obtained under truly perfect linkage.
\end{abstract}

Key words: Population size estimator; undercoverage; probabilistic record linkage.

\section{Introduction}

Capture-recapture methodology goes back to at least the ecological setting of estimating the size of fish and wildlife populations. The basic idea is to take a first sample (capture), tag or mark the captured animals, return them to their population and take a second sample (recapture). Among the recaptures, some of the animals will be marked, others not. The relation between the tagged and non-tagged animals in the second sample is used to construct an estimate of the total population size. See for example Petersen (1896) and Lincoln (1930). Since then, it has been used not only to estimate animal population sizes, but also to estimate undercount in traditional censuses (for an overview see e.g., Fienberg 1992). More recently, it was used to estimate the undercoverage of registers used for the Dutch Census (Gerritse et al. 2016a).

${ }^{1}$ Statistics Netherlands, P.O.Box 24500, 2490 HA The Hague, The Netherlands. Emails: pp.dewolf@cbs.nl,dj.vanderlaan@cbs.nl, and db.zult@cbs.nl.

Acknowledgments: The authors like to thank Jeroen Pannekoek for reviewing an earlier version of the article. The views expressed in this article are those of the authors and do not necessarily reflect the policy of Statistics Netherlands. 
In the original setting, one of the assumptions is that the units can be classified without error to belong to the first sample only the second sample only or the overlap of the two samples. This assumption was likely to be met, when the marking of the units in the first sample would stick to the animals during the second sampling (no tag-loss). In the setting of estimating the undercount of a register, this assumption is translated to the assumption that units in the two registers can be linked without error, that is, all links are found and no erroneous links are established. With linking two records, we mean deciding that those records represent the same population unit. Whenever the both registers contain the same reliable unique identifiers like a social security number, it is likely that this assumption holds. However, not all registers contain such a uniform unique identifier. Actually, when considering undercoverage of registers, one can not rely on the existence of such unique identifiers only. Indeed, in order to find units that are not properly registered one should also use sources that do not have such a unique identifier for all units.

In case when a unique identifier is not available, one often relies on probabilistic record linkage techniques like the one developed in Fellegi and Sunter (1969). In this setting, the assumption of perfect linkage is not likely to be met in practice. Especially in a large population, two individuals might, for example, have the same name, leading to a false link, or one individual might be known under two different names, leading to a missed link. This last case would be identical to tag-loss in a classical capture-recapture setting, while the first case can only occur when tags or id-codes can be passed around within the population of interest.

In the presence of linkage errors, the standard capture-recapture estimate of the unknown population size can be biased see e.g., Gerritse et al. (2016b). In Ding and Fienberg (1994) the standard capture recapture estimator is adjusted to correct for linkage errors. In that paper, they considered the situation where a post-enumeration survey (PES) is used to estimate the undercoverage of the population census. See for example, Wolter (1986) for an explanation of using a PES. Ding and Fienberg (1994) assume that the false match that affects the population size estimator mostly occurs when a record from the subset of the PES that should not be matched is actually linked to a record from the subset of the census that should not be matched. In other words, they assume a one-way linkage error, linking PES records to census records. Moreover, they assume that all records in the PES will be linked to a record in the census. Cadwell et al. (2005) also correct for linkage errors, but they use the concept of 'potential linkage' in a bootstrap procedure to construct a population size interval. Their method is potentially interesting when something like a PES is not available.

Di Consiglio and Tuoto (2015) argue that in the setting of administrative data sources, a one-way linkage direction is not guaranteed. That is, they allow for the possibility that a population unit residing in one administrative data source, but not in the other, can be (incorrectly) linked to a unit in the other administrative data source irrespective of which data source is called 'the one' and which is called 'the other'. Hence, they propose a twoway correction for linkage error. In their paper, they assume that the probability of a false match is equal in both linkage directions. We will call this the symmetric two-way correction for linkage error. Using the same error probability in both directions is appropriate in case the two administrative data sources are (approximately) of equal size. 
When two registers differ considerably in size, a further extension would be to allow for different error probabilities in the two linkage directions. This would be even more evident when (forced) one-to-one linkage is used. One-to-one linkage means that a record from one source is allowed to be linked to one and only one record from the other source. Since the largest source contains units that are not present in the smaller source, in case of one-to-one linkage a subset of those units can never be linked; there are simply not enough 'target' records in the smaller source. Records that will never be linked, will also never be linked incorrectly. In other words, a unit in the largest administrative data source has a smaller chance of being falsely linked with a unit in the smaller administrative data source, compared to the other way around. Thus, in the current article we introduce an asymmetric two-way correction for linkage error. The formulation of this asymmetric two-way corrections has three parameters. Choosing specific values for those parameters, the formula can cover the one-way correction and the symmetric two-way correction as well.

The outline of the article is as follows. We start by explaining the general setting of capture-recapture and probabilistic linkage. In Section 3 we briefly present the noncorrected estimator, the one-way corrected estimator, the symmetric two-way corrected estimator and our asymmetric two-way corrected estimator. The formula of the asymmetric two-way correction can be viewed as a general estimator, in the sense that all introduced estimators can be expressed with this formula. Finally, we unify all estimators by choosing specific 'optimal' parameters. The following section, Section 4, shows some simulation results using publicly-available fictitious data on the UK population census. In Section 5 we draw conclusions and the appendices contain some technical details. Section 6 contains Appendices 1-4.

\section{General Setting}

Let us first introduce the notation that will be used throughout the remainder of this article. We try to stay close to the notation used in Ding and Fienberg (1994) and Di Consiglio and Tuoto (2015). We also present the general assumptions underlying the capture-recapture methodology when applied with two registers. Note that we will only discuss the situation of two registers that are linked using probabilistic record linkage methods.

\subsection{Capture-Recapture with Two Registers}

Let $R_{1}$ and $R_{2}$ denote two registers containing units from a common population $\mathcal{X}$ of unknown size $N_{\mathcal{X}}$. Assuming we can identify population units to belong to either one or both of the registers, we get Table 1 and Table 2 . In Table 1 the numbers correspond to the unobservable true population counts, whereas the numbers in Table 2 are the observed counts after the linkage process has taken place and thus depend on the used linkage procedure.

In the tables, the first subscript denotes whether or not a population unit resides in $R_{1}$ and the second subscript denotes whether or not a population unit resides in $R_{2}$. So, for example, $N_{10}$ denotes the (unobserved) number of population units that resides in $R_{1}$ but not in $R_{2}$. Note that, assuming no duplicates in each $R_{i}, n_{1+}=N_{1}$ is the size of $R_{1}$ and $n_{+1}=N_{2}$ is the size of $R_{2}$. Moreover, $N_{\neg i}$ denotes the number of units in the population 
Table 1. Counts based on population.

\begin{tabular}{llll|l}
\hline & \multicolumn{5}{c}{ Unit in $R_{2}$} \\
& & yes & no \\
Unit in $R_{1}$ & yes & $N_{11}$ & $N_{10}$ & $N_{1}$ \\
& no & $N_{01}$ & $N_{00}$ & $N_{\neg 1}$ \\
\cline { 3 - 5 } & & $N_{2}$ & $N_{\neg 2}$ & $N_{\mathcal{X}}$ \\
\hline
\end{tabular}

Table 2. Counts based on linkage process.

\begin{tabular}{llll|l}
\hline & & \multicolumn{3}{c}{ Unit in $R_{2}$} \\
& yes & no \\
& yes & $n_{11}$ & $n_{10}$ & $n_{1+}$ \\
Unit in $R_{1}$ & no & $n_{01}$ & 0 & $n_{01}$ \\
\cline { 3 - 5 } & & $n_{+1}$ & $n_{10}$ & $n_{++}$ \\
\hline
\end{tabular}

that do not reside in $R_{i}$, that is, $N_{\neg 1}=N_{\mathcal{X}}-N_{1}$ and $N_{\neg 2}=N_{\mathcal{X}}-N_{2}$. Even after the linkage process has taken place, we still cannot observe population units that are included in neither register (i.e., $N_{00}$ ). That means that $N_{\neg 1} \geq n_{01}$ and $N_{\neg 2} \geq n_{10}$.

Using similar notation, we can write the probability that a population unit resides in register $R_{i}$ as $p_{i}$, and decompose those probabilities as follows: $p_{1}=p_{11}+p_{10}$ and $p_{2}=p_{11}+p_{01}$ where $p_{11}$ denotes the probability that a unit resides in both registers, $p_{10}$ the probability that a unit does reside in $R_{1}$ but not in $R_{2}$ and $p_{01}$ the probability that a unit resides in $R_{2}$ but not in $R_{1}$.

The general assumptions in capture-recapture estimation are:

- The population $\mathcal{X}$ is closed, that is, units can neither enter nor leave the population during the capture-recapture experiment.

- There are no erroneous captures, that is, only units from $\mathcal{X}$ can be captured.

- There are no duplicates in either register, that is, units can only be captured once per register.

- The event that a unit resides in $R_{1}$ is independent of the event that a unit resides in $R_{2}$.

- The probability that a unit resides in $R_{i}$ is the same for all units in $\mathcal{X}$.

- There is no error in allocating the units to $R_{1}, R_{2}$ or both.

These assumptions imply that $N_{11} / N_{1}=N_{2} / N_{\mathcal{X}}$ or equivalently, $N_{\mathcal{X}}=\left(N_{1} N_{2}\right) / N_{11}$. Hence, under perfect conditions a natural estimator would be the one introduced in Petersen (1896): $\hat{N}_{\mathcal{X}}=\left(n_{1+} n_{+1}\right) / n_{11}$. See Subsection 3.1 as well.

\subsection{Probabilistic Record Linkage}

The probabilistic record linkage technique we will assume in this article is the one described in Fellegi and Sunter (1969). In their approach, they consider the set of all 
possible pairs $(a, b)$ of records from $R_{1}$ and $R_{2}:\left\{(a, b) \mid a \in R_{1}\right.$ and $\left.b \in R_{2}\right\}$. They decompose that set into two disjunct sets. Set $\mathcal{M}$ consisting of all pairs of records of matches and set $\mathcal{U}$ of all pairs of records of nonmatches. Hence, for example, a pair $(a, b)$ in the set $\mathcal{U}$ of nonmatches should consist of a record $a$ from register $R_{1}$ and a record $b$ from $R_{2}$ where $a$ and $b$ refer to two different population units. See Figure 2 in Appendix 1 (Subsection 6.1) for a graphical representation of the sets $\mathcal{M}$ and $\mathcal{U}$.

Fellegi and Sunter then describe a model to decide whether an observed pair of records should be allocated to $\mathcal{M}$ or to $\mathcal{U}$. To that end they use comparison functions that assign a value to a pair indicating the amount of similarity between the two records. For example, in case of personal data, a comparison function could assign a value zero whenever the name of the person of record $a$ is not exactly equal to the name of the person of record $b$, and a value of one whenever the names are exactly equal. Obviously, this can be more elaborate assigning a value between zero and one in case of small spelling mistakes. Different comparison functions can be applied to different variables within a record, which would result in a comparison vector.

Selecting a pair of records at random from all possible pairs, the comparison function applied to that selected pair is a random variable. They define the $m$-probability as the probability that a certain value of the comparison function is found among a pair of records that should belong to the set $\mathcal{M}$ of matches and the $u$-probability as the probability that a certain value of the comparison function is found among a pair of records that should belong to the set $\mathcal{U}$ of nonmatches. Using those probabilities, they assign weights to each possible pair and say that a pair of records is linked whenever the weight is above some threshold and not linked whenever that weight is below that threshold. Since this is defined at the level of pairs of records, it is possible that several records from register $R_{1}$ are said to be linked to the same record in register $R_{2}$; whenever a pair has a weight above the threshold, it will be said to be linked. In practice, often a one-to-one linkage is then enforced. One of those pairs is selected and designated to be a link, while the other pairs are considered to be non-links despite their weight being above the threshold.

In their paper, Fellegi and Sunter (1969) consider two error probabilities; the probability of a false link (assigning a pair of records to $\mathcal{M}$ where it should be assigned to $\mathcal{U}$ ) and the probability of a false non-link (assigning a pair of records to $\mathcal{U}$ where it should be assigned to $\mathcal{M}$ ). Note that these probabilities are thus defined at the level of pairs of records and not on the level of individual records. In the description of the correction methods (see Section 3) error probabilities are defined at the level of individual records. To be able to discuss the correction methods for linkage error, it is convenient to decompose our registers $R_{i}$ each into two disjunct sets $M_{i}$ and $U_{i}$. Now $M_{i}$ consists of all unique records from register $R_{i}$ that should appear in a pair of matches and $U_{i}$ of all other unique records from register $R_{i}$. Figure 2 in Appendix 1 (Subsection 6.1) graphically shows the differences between the sets $\mathcal{M}, \mathcal{U}, M_{i}$ and $U_{i}$.

Linking registers with many records would lead to a huge number of pairs. Under these circumstances a technique known as blocking is often used to improve efficiency. With blocking, the registers are split into subsets that agree on one or more highly discriminating identifiers and the linkage process is applied within each subset separately. For the sake of simplicity, we will not address the use of blocking in the current article, since blocking would affect the (estimation of the) $m$-probabilities in a complex way. 


\section{Estimation of the Population Size}

In this section, we will first briefly present the existing estimators for the population size under no linkage error, one-way error correction and symmetric two-way error correction. At the end of this section, we will introduce our new asymmetric two-way error correction estimator.

Using the notation from Subsection 2.1, we assume that the number of individuals that fall in the four interior cells of Table 2 have a multinomial distribution

$$
\left(n_{11}, n_{10}, n_{01}, N_{\mathcal{X}}-n_{++}\right) \sim \operatorname{Mult}\left(N_{\mathcal{X}}, p_{11}, p_{10}, p_{01}, p_{00}\right)
$$

where $n_{++}=\mathrm{n}_{11}+n_{10}+n_{01}$. Like in Ding and Fienberg (1994), we will derive the estimators using the approach of maximizing the conditional likelihood as described in Sanathanan (1972). In that approach, the likelihood is written as a product of two likelihoods $L_{1}(\cdot)$ and $L_{2}(\cdot)$, where $L_{1}(\cdot)$ is the likelihood of $\left(n_{11}, n_{10}, n_{01}\right)$ for fixed $n_{++}$and $L_{2}(\cdot)$ the likelihood of $n_{++}$, given the cell-probabilities $p_{11}, p_{10}$ and $p_{01}$. In the conditional approach, first $L_{1}(\cdot)$ is maximized to derive the maximum likelihood (ML) estimates of the cell probabilities, after which the $N_{\mathcal{X}}$ is found that maximizes $L_{2}(\cdot)$, given the values of $p_{11}, p_{10}$ and $p_{01}$.

Using that $\mathbb{E}\left(n_{++}\right)=\mathbb{E}\left(n_{1+}\right)+\mathbb{E}\left(n_{+1}\right)-\mathbb{E}\left(n_{11}\right)=\left(p_{1}+p_{2}-p_{11}\right) N_{\mathcal{X}}$, where $\mathbb{E}$ denotes taking expectation, we derive the following generic formulation of an estimator of the population total

$$
\hat{N}_{\chi}=\frac{n_{++}}{\hat{p}_{1}+\hat{p}_{2}-\hat{p}_{11}}
$$

In the following subsections we will derive conditional ML estimators of the cell probabilities under different linkage error scenarios.

\subsection{No Linkage Error}

Under independence and perfect linkage, we would have the following equations for the probabilities of recording population units in the different observed counts $n_{i j}$ :

$$
\begin{aligned}
& p_{11}=p_{1} p_{2} \\
& p_{10}=p_{1}-p_{11}=p_{1}\left(1-p_{2}\right) \\
& p_{01}=p_{2}-p_{11}=p_{2}\left(1-p_{1}\right)
\end{aligned}
$$

Using the conditional ML approach we would get the estimators

$$
\hat{p}_{1}=\frac{n_{11}}{n_{+1}} \quad \text { and } \quad \hat{p}_{2}=\frac{n_{11}}{n_{1+}}
$$

Plugging those estimators into (2) and (1), the estimator of the population total then becomes after some straightforward calculations

$$
\hat{N}_{\mathcal{X}}^{P}=\frac{n_{1+} n_{+1}}{n_{11}}
$$

and this is essentially the estimator as described in for example, Petersen (1896). 


\subsection{One Way Correction (OC)}

In Ding and Fienberg (1994) the situation of linkage error is considered under the assumptions that (using the notation as in Subsection 2.2)

(a) A true match between records from $M_{1}$ and $M_{2}$ remains a match with probability $0<\alpha \leq 1$.

(b) A record from $M_{1}$ is matched incorrectly with a record in $M_{2}$ with negligible probability.

(c) A false match between records from $M_{1}$ and $U_{2}$ occurs with negligible probability.

(d) A false match between records from $U_{1}$ and $M_{2}$ occurs with negligible probability.

(e) Each record from $U_{1}$ will be linked with a record in $U_{2}$ with common probability $0 \leq \beta<1$.

The reason for assuming negligible probabilities for (b), (c) and (d) is that in those cases, two errors are made; both the correct match is not made and an incorrect match is made. Cases (a) and (e) are each related to making only one error: in case of (a) with probability $1-\alpha$ only a correct match is missed and in case of (e) only an incorrect match is made.

The just introduced probabilities $\alpha$ and $\beta$ are defined at record level. Note that the probabilities in the Fellegi and Sunter (1969) setting (see Subsection 2.2), sometimes also denoted by $\alpha$ and $\beta$, are defined at the level of pairs of records and are thus fundamentally different from the ones used in the current article. Moreover, note that a large $\alpha$ implies more missed links (in expectation), which in turn leads to an upward bias in the estimator $\hat{N}_{\mathcal{X}}$. A large $\mathrm{S}$ implies more false links (in expectation), which would lead to a downward bias in $\hat{N}_{\mathcal{X}}$.

Under the aforementioned assumptions we get the following relations

$$
\begin{aligned}
& p_{11}=\alpha p_{1} p_{2}+\beta p_{1}\left(1-p_{2}\right) \\
& p_{10}=p_{1}-p_{11}=p_{1}-\alpha p_{1} p_{2}-\beta p_{1}\left(1-p_{2}\right) \\
& p_{01}=p_{2}-p_{11}=p_{2}-\alpha p_{1} p_{2}-\beta p_{1}\left(1-p_{2}\right)
\end{aligned}
$$

Note that the 'one-way' correction is reflected in (6); the second term on the right hand side only shows the probability of falsely linking $(\beta)$ a unit that resides in $R_{1}\left(p_{1}\right)$ but not in $R_{2}\left(1-p_{2}\right)$. The probability of falsely linking a unit that resides in $R_{2}$ but not in $R_{1}$ is not considered, that is, only one linkage direction is considered.

The conditional ML estimators are then given by (Ding and Fienberg 1994)

$$
\hat{p}_{1}=\frac{n_{11}-\beta n_{1+}}{(\alpha-\beta) n_{+1}} \quad \text { and } \quad \hat{p}_{2}=\frac{n_{11}-\beta n_{1+}}{(\alpha-\beta) n_{1+}}
$$

Plugging this into (6) and (1), the population total then can be estimated by

$$
\hat{N}_{\mathcal{X}}^{O C}=\frac{(\alpha-\beta) n_{11}}{n_{11}-\beta n_{1+}} \frac{n_{1+} n_{+1}}{n_{11}}=\frac{(\alpha-\beta) n_{11}}{n_{11}-\beta n_{1+}} \hat{N}_{\chi}^{P}
$$

with $\hat{N}_{\mathcal{X}}^{P}$ as defined in (5). Note that this estimator depends on the parameters $\alpha$ and $\beta$ which are unknown in practice and should therefore be estimated. This will be discussed in Subsection 3.5. 


\subsection{Symmetric Two-Way Correction (SC)}

In Di Consiglio and Tuoto (2015) it is proposed to relax the assumption of the one-way correction and to allow a two-way correction. This means that assumption (e), as given in the description of the one-way correction, is relaxed to allow for a unit in $U_{1}$ that is not in $U_{2}$ still to be (incorrectly) linked to a unit in $U_{2}$ as well as to allow for a unit in $U_{2}$ that is not present in $U_{1}$ still to be (incorrectly) linked to a unit in $U_{1}$. Both events occur with the same probability $0 \leq \beta<1$.

This results in the following equations

$$
\begin{aligned}
& p_{11}=\alpha p_{1} p_{2}+\beta p_{1}\left(1-p_{2}\right)+\beta p_{2}\left(1-p_{1}\right) \\
& p_{10}=p_{1}-p_{11}=p_{1}-\alpha p_{1} p_{2}-\beta p_{1}\left(1-p_{2}\right)-\beta p_{2}\left(1-p_{1}\right) \\
& p_{01}=p_{2}-p_{11}=p_{2}-\alpha p_{1} p_{2}-\beta p_{1}\left(1-p_{2}\right)-\beta p_{2}\left(1-p_{1}\right)
\end{aligned}
$$

Again, under certain regularity conditions and using the conditional likelihood approach, they derive that the ML estimators are then given by

$$
\hat{p}_{1}=\frac{n_{11}-\beta\left(n_{1+}+n_{+1}\right)}{(\alpha-2 \beta) n_{+1}} \quad \text { and } \quad \hat{p}_{2}=\frac{n_{11}-\beta\left(n_{1+}+n_{+1}\right)}{(\alpha-2 \beta) n_{1+}}
$$

Plugging this into (10) and (1), the population total can then be estimated by

$$
\hat{N}_{\mathcal{X}}^{S C}=\frac{(\alpha-2 \beta) n_{11}}{n_{11}-\beta\left(n_{1+}+n_{+1}\right)} \frac{n_{1+} n_{+1}}{n_{11}}=\frac{(\alpha-2 \beta) n_{11}}{n_{11}-\beta\left(n_{1+}+n_{+1}\right)} \hat{N}_{\mathcal{X}}^{P}
$$

\subsection{Asymmetric Two-Way Correction (AC)}

As a further relaxation of the assumptions, we propose to allow for different probabilities of false links. This means that we allow for a unit present in $U_{1}$ but not present in $U_{2}$ to be linked to a unit in $U_{2}$ with probability $0 \leq \beta_{1}<1$ and a unit present in $U_{2}$ but not present in $U_{1}$ to be linked to a unit in $U_{1}$ but with probability $0 \leq \beta_{2}<1$.

Now the equations for the probabilities of recording population units in the different observed counts become

$$
\begin{aligned}
& p_{11}=\alpha p_{1} p_{2}+\beta_{1} p_{1}\left(1-p_{2}\right)+\beta_{2} p_{2}\left(1-p_{1}\right) \\
& p_{10}=p_{1}-p_{11}=p_{1}-\alpha p_{1} p_{2}-\beta_{1} p_{1}\left(1-p_{2}\right)-\beta_{2} p_{2}\left(1-p_{1}\right) \\
& p_{01}=p_{2}-p_{11}=p_{2}-\alpha p_{1} p_{2}-\beta_{1} p_{1}\left(1-p_{2}\right)-\beta_{2} p_{2}\left(1-p_{1}\right)
\end{aligned}
$$

Under certain regularity conditions, we then get the following ML estimators

$$
\hat{p}_{1}=\frac{n_{11}-\beta_{1} n_{1+}-\beta_{2} n_{+1}}{\left(\alpha-\left(\beta_{1}+\beta_{2}\right)\right) n_{+1}} \quad \text { and } \quad \hat{p}_{2}=\frac{n_{11}-\beta_{1} n_{1+}-\beta_{2} n_{+1}}{\left(\alpha-\left(\beta_{1}+\beta_{2}\right)\right) n_{1+}}
$$

See Appendix 2 (Subsection 6.2) for a discussion on admissibility to obtain proper values for the probabilities $\hat{p}_{1}$ and $\hat{p}_{2}$ in the interval $[0,1]$. 
Plugging (17) into (14) and (1), the population total can then be estimated by

$$
\hat{N}_{\mathcal{X}}^{A C}=\frac{\left(\alpha-\left(\beta_{1}+\beta_{2}\right)\right) n_{11}}{n_{11}-\beta_{1} n_{1+}-\beta_{2} n_{+1}} \frac{n_{1+} n_{+1}}{n_{11}}=\frac{\left(\alpha-\left(\beta_{1}+\beta_{2}\right)\right) n_{11}}{n_{11}-\beta_{1} n_{1+}-\beta_{2} n_{+1}} \hat{N}_{\mathcal{X}}^{P}
$$

Note that this formulation covers all previous situations by choosing appropriate $\alpha, \beta_{1}$ and $\beta_{2}$

- Petersen estimator: $\alpha=1$ and $\beta_{1}=\beta_{2}=0$

- One-way correction: $\alpha=\alpha, \beta_{1}=\beta$ and $\beta_{2}=0$

- Symmetric two-way correction: $\alpha=\alpha, \beta_{1}=\beta_{2}=\beta$

\subsection{Linking the Correction Methods}

We consider the Petersen estimator in case of perfect linkage, that is, knowing the true $N_{1}$, $N_{2}$ and $N_{11}$, the 'optimal' estimator and call it the 'true Petersen estimator' (TP)

$$
N_{\mathcal{X}}^{T P}=\frac{N_{1} N_{2}}{N_{11}}=\frac{n_{1+} n_{+1}}{N_{11}}
$$

Equating the AC estimator to the TP estimator, that is, setting $\hat{N}_{\mathcal{X}}^{A C}=N_{\mathcal{X}}^{T P}$, we get the following relationship between the parameters

$$
\alpha N_{11}+\beta_{1}\left(N_{1}-N_{11}\right)+\beta_{2}\left(N_{2}-N_{11}\right)=\alpha N_{11}+\beta_{1} N_{10}+\beta_{2} N_{01}=n_{11}
$$

Note that the left-hand side equals the expected number of links under the model for linkage error.

Let us first explore this relationship under the unrealistic assumption that we know the true $N_{11}$. A natural choice for the parameter $\alpha$ would then be the fraction of true population matches among the links from the linkage process. We will denote this natural choice by $\check{\alpha}$. Substituting that natural choice in (19) and setting $\beta_{1}=\beta^{O C}$ and $\beta_{2}=0$, we get

$$
\alpha^{O C}=\check{\alpha}=\frac{m_{11}}{N_{11}} \quad \text { and } \quad \beta^{O C}=\frac{n_{11}-m_{11}}{N_{1}-N_{11}}
$$

where $m_{11}$ is the number of true population matches among the links from the linkage process. We will call this choice of parameters the optimal OC-parameters.

In case of the symmetric two-way correction, using the natural choice for $\alpha$ and setting $\beta_{1}=\beta_{2}=\beta^{S C}$ leads to

$$
\alpha^{S C}=\check{\alpha}=\frac{m_{11}}{N_{11}} \quad \text { and } \quad \beta^{S C}=\frac{n_{11}-m_{11}}{N_{1}+N_{2}-2 N_{11}}
$$

We will call this choice of parameters the optimal SC-parameters.

In case of the asymmetric two-way correction, we need an additional constraint to uniquely define 'optimal AC-parameters'. In practice, it is convenient to enforce one-toone linkage in the process. Under that assumption, we can derive the following relationship between the parameters of the asymmetric two-way estimator (see the Appendix 3, Subsection 6.3, for a derivation)

$$
\beta_{1}=\frac{\left(\alpha n_{+1}-n_{11}\right) \beta_{2}}{\left(\alpha n_{1+}-n_{11}\right)-2 \beta_{2}\left(n_{1+}-n_{+1}\right)}
$$


In case we want to satisfy both (20) and (19) using the natural $\check{\alpha}$ parameter, we get either

$$
\alpha^{A C}=\check{\alpha}=\frac{m_{11}}{N_{11}}, \quad \beta_{1}^{A C}=\frac{n_{11}-m_{11}}{2\left(N_{1}-N_{11}\right)} \quad \text { and } \quad \beta_{2}^{A C}=\frac{n_{11}-m_{11}}{2\left(N_{2}-N_{11}\right)}
$$

or

$$
\tilde{\alpha}^{A C}=\check{\alpha}=\frac{m_{11}}{N_{11}}, \quad \tilde{\beta}_{1}^{A C}=\frac{m_{11} N_{2}-n_{11} N_{11}}{m_{11}\left(N_{2}-N_{1}\right)} \quad \text { and } \quad \tilde{\beta}_{2}^{A C}=\frac{m_{11} N_{1}-n_{11} N_{11}}{m_{11}\left(N_{1}-N_{2}\right)}
$$

where $m_{11}$ again is the number of true population matches among the links from the linkage process. For the second set of parameters $\left(\tilde{\alpha}^{A C}, \tilde{\beta}_{1}^{A C}\right.$ and $\left.\tilde{\beta}_{2}^{A C}\right)$ it holds that the $\tilde{\beta}$ 's will be undefined in case $N_{1}=N_{2}$. Moreover, when $N_{1} \neq N_{2}$, one of them will be negative, which contradicts the fact that the $\tilde{\beta}$ 's are probabilities. We will hence call the first set of parameters the optimal AC-parameters. Note that, in case register $R_{1}$ is the largest and hence under one-to-one linkage $N_{1}-N_{11}>N_{2}-N_{11}$, we get $\beta_{1}^{A C}<\beta_{2}^{A C}$ as expected (see discussion in introduction).

According to the error correction model, a false match between a record from $U_{1}$ with a record from $U_{2}$ occurs with probability $\beta_{1}$ and, independently, a false match between a record from $U_{2}$ with a record from $U_{1}$ occurs with probability $\beta_{2}$. Considering these events independently, we would count such a link twice. However, enforcing one-to-one linkage, these two events can only happen at the same time. This is reflected in the factor $1 / 2$ in the optimal AC-parameters $\beta_{i}^{A C}$.

Given the true $N_{11}$ and choosing the parameters such that they satisfy Equation (19) would thus lead to the optimal estimator. Indeed, using the optimal OC-parameters, the optimal SC-parameters or the optimal AC-parameters will all yield the same estimator, that is, the true Petersen estimator TP (with perfect linkage).

Unfortunately, in practice we do not know the true $N_{11}$. Hence, we need to estimate the $\alpha$ and $\beta_{i}$ parameters. As long as the estimated parameters satisfy relation (19), the resulting estimates will be exactly the same for all estimators. This would, for example, be the case when we would estimate the optimal parameters by plugging in some estimate for $N_{11}$, since $N_{1}, N_{2}, n_{11}$ and $m_{11}$ are the same in all settings. Indeed, the resulting estimators would then be given by the simple formula

$$
\hat{N}_{\mathcal{X}}=\frac{N_{1} N_{2}}{\hat{N}_{11}}
$$

where $\hat{N}_{11}$ is a (consistent) estimator of the 'true' overlap between the two registers.

Another possibility would be to use a sample of one of the registers and determine the true matches for that sample. Dividing that number by the sampling fraction would yield a direct estimate of $N_{11}$. Similarly, we could obtain direct estimates of $n_{11}$ and $m_{11}$. Note that a direct estimate of $n_{11}$ is needed instead of the original $n_{11}$ to prevent the estimated $m_{11}$ becoming larger than the original $n_{11}$.

Yet another approach would be to use expert knowledge on the linkage errors, for example, asking experts to give estimates of the parameters. In that case, these expert guesses would not necessarily satisfy relation (19) and the estimators could thus yield different values. 


\section{Simulation Study}

The evaluation of a linkage error correction method in a capture-recapture setting requires knowledge on the bias introduced by the linkage errors. In real data applications the bias is obviously usually unknown. However, in case of a fictitious population the bias can be determined accurately. Such a fictitious population may be a real or simulated list of individuals that is then defined as the 'true population'. Subsets can then be randomly selected from this list and linked with and without linkage error. This way capturerecapture can be applied with and without linkage error, as well as with and without linkage error correction. Hence, the different linkage error correction methods can be evaluated. Moreover, this procedure can be repeated multiple times with different randomly selected subsets in order to obtain multiple estimates. As a result, the linkage error bias and variance over the repeated estimates can be assessed.

We used the same data set as used by Di Consiglio and Tuoto (2015). This publicly available data set concerns a fictitious population that is based on the UK population census, as created for the ESSnet DI (McLeod et al. 2011). The ESSnet DI was a European project on data integration (Record Linkage, Statistical Matching, Micro Integration Processing), running from 2009 to 2011 . The advantage of this data set is twofold. Firstly, it is publicly available, which supports reproducibility of our simulation study. Secondly, it is designed to resemble the situation a researcher might encounter in real life. For instance, the samples contain errors such as typical spelling errors in names or incorrect house numbers. Moreover, it is unclear (to us) how exactly the population samples were generated; for example did some groups have higher probability to be in certain samples or not? The main methodological difference between a real data set and this fictitious population is that the true population size is known, while most other real data issues remain present.

Since ensuring that the parameter estimates satisfy relation (19) will result in the same estimates of the population size for all estimators introduced in Section 3, we will not explicitly compare them. This approach deviates from Di Consiglio and Tuoto (2015), where the estimator based on the SC model is compared to the estimator based on the OC model. They conclude that the SC estimator outperforms both the Petersen and the OC estimator. Their conclusion that the SC estimator, as well as the OC estimator, outperform the Petersen estimator in case of linkage errors is fully justified. However, their result that the SC estimator outperforms the OC estimator seems to contradict our findings in Section 3. This apparent contradiction can be explained by the fact that in Di Consiglio and Tuoto (2015) the parameters $\alpha^{S C}$ and $\beta^{S C}$ (i.e., the optimal parameters under the SC model) are used in both the SC and OC estimator. We now know that in case of the OC estimator the optimal parameters are $\alpha^{O C}$ and $\beta^{O C}$ and therefore should have been chosen differently.

In our simulation study, we concentrate on different ways to estimate the parameters instead of comparing the different estimators with one choice of parameter estimates. We will use different methods to estimate $N_{11}$ and $m_{11}$ and plug those estimates into the formulas of our 'optimal' parameters, to show empirically that these estimates indeed lead to the same estimate of the population total and to study the behaviour of that estimate with respect to its bias and variance. 


\subsection{Setup}

From the ESSnet DI data we used the files Person (a list of persons, acting as the population), CIS (observations from a Customer Information System, being a combination of tax and benefit data) and PRD (observations from the Patient Register Data of the National Health Service). The Person dataset is comprised of 26,625 individuals, the CIS has a coverage probability of that population of $\tau_{1}=0.924$ and the PRD of $\tau_{2}=0.930$.

To reduce computation time and to be able to apply the linkage process without blocking, we repeatedly constructed a smaller population and corresponding registers from those files, using the following steps

1. Draw a simple random sample without replacement of size 10,000 from Person. This will be our population $\mathcal{X}$ with size $N_{\mathcal{X}}$.

2. Select the records from CIS that are present in population $\mathcal{X}$ to get register $R_{1}$.

3. Select the records from PRD that are present in population $\mathcal{X}$. Randomly select a fraction $f_{2}$ of those records to get register $R_{2}$.

This way we obtained multiple instances of a population and the corresponding registers, where one of them covers the population for about $92.4 \%$ and the other for about $f_{2}$ times $93.0 \%$. Note that, for small values of $f_{2}$, the two registers differ substantially in size.

In the above mentioned setup, one of the registers is quite close in size to the total population: $R_{1}$ covers the population for about $92.4 \%$. This resembles the situation where an NSI has a high quality population register and wants to quantify the quality of that register in terms of estimated undercoverage. A different situation may be that no such high quality register exists. In that case neither register used in the capture-recapture procedure has high coverage. To get an idea how the error correction methods work in that case we included two additional scenarios. We maintained the small coverage of $R_{2}$ (using $f_{2}=0.15$ ) but additionally we reduced the coverage of $R_{1}$ by randomly selecting a fraction $f_{1}$ of its records, where $f_{1} \in\{0.15,0.5\}$. Table 3 shows the mean coverage of the registers we obtained in our simulations.

In Di Consiglio and Tuoto (2015) several linkage scenarios were mentioned: a bronze, a silver and a gold scenario. In the current paper we will only use their silver scenario, that is, we only use the full date of birth (day (DB_D), month (DB_M) and year (DB_Y)) as key variables in the linkage process. We have chosen the silver scenario because it allows for two types of linkage error. Firstly, two different individuals may have the same date of birth and therefore may be falsely linked. Secondly, due to some measurement errors, an individual that is in both samples may be falsely not linked.

Table 3. Mean coverage $\bar{\tau}_{\mathrm{i}}$ of register $R_{i}$ over the 100 replications.

\begin{tabular}{lccc}
\hline$f_{1}$ & $f_{2}$ & $\bar{\tau}_{1}$ & $\bar{\tau}_{2}$ \\
\hline 1.00 & 0.90 & $92.42 \%$ & $83.65 \%$ \\
1.00 & 0.50 & $92.46 \%$ & $46.48 \%$ \\
1.00 & 0.15 & $92.44 \%$ & $13.93 \%$ \\
0.50 & 0.15 & $46.23 \%$ & $13.94 \%$ \\
0.15 & 0.15 & $13.87 \%$ & $13.94 \%$ \\
\hline
\end{tabular}


Names and surnames would have been better discriminating identifiers, but in the absence of those variables (e.g., due to privacy restrictions), the full date of birth is still reasonably well discriminating.

For the comparison function of the probabilistic record linkage process (see Subsection 2.2), we simply used 'equality' on all key variables separately. That is, whenever two records $a$ and $b$ are compared, the comparison function for key variable $V_{i}$ is 1 when $V_{i}(a)=V_{i}(b)$ or 0 when $V_{i}(a) \neq V_{i}(b)$. Whenever $V_{i}$ is missing in at least one of the two records, the comparison function is defined to be 0 as well. To perform the probabilistic record linkage as described in Subsection 2.2, we used our own R-code. In that code we also forced one-to-one linkage. (See https://github.com/djvanderlaan/reclin for the R-package reclin that we used.)

We implemented four methods to obtain values for the $N_{11}, m_{11}$ and $n_{11}$ needed in the formulas for the 'optimal' parameters.

A Since we use simulated data, we know the true $m_{11}$ and $N_{11}$ by design. The $n_{11}$ follows from the linkage process.

B Using the EM-algorithm (see e.g., Herzog et al. 2007) on the complete registers to estimate the posterior $m$-probabilities. Those posterior probabilities were used to estimate the $m_{11}$ and $N_{11}$. The $n_{11}$ follows from the linkage process.

C Using a sample of size 200 from the smallest register of which we determine the true match-status. Using that sample, we fitted a logistic model (see the Appendix 4 (Subsection 6. 4) for more information on the used model) and used that to predict the $m$-probabilities for the complete registers. Those posterior probabilities were used to estimate the $m_{11}$ and $N_{11}$. The $n_{11}$ follows from the original linkage process.

D Using a sample of size 200 from the smallest register of which we determine the true match-status. Using that sample we calculated the direct estimates of $n_{11}, N_{11}$ and $m_{11}$ for the complete registers.

In methods $\mathrm{B}$ and $\mathrm{C}$, summing the posterior $m$-probabilities over all linked pairs yields an estimate of $\mathrm{m}_{11}$, whereas summing those probabilities over all possible pairs yields an estimate of $N_{11}$. For a definition of posterior $m$-probabilities and why summing them is appropriate, we refer to Fellegi \& Sunter (1969). Methods B, C and D serve to illustrate how information available during an actual record linkage process can be used to correct the estimator for linkage errors. As long as the sample used in methods C and D is a representative sample of possible record pairs, these methods should give unbiased estimates of $N_{11}, m_{11}$ and $n_{11}$. Other methods or refinements of these methods that might give more precise estimates are possible. However, finding such refinements is not the main focus of this article; we want to show that even relatively simple methods can already correct for bias due to linkage errors.

With those estimated sizes, we then used the formulas for the 'optimal' parameters as derived in Subsection 3.5 to get estimates of the population size. As discussed in that section, we should then obtain exactly the same estimates for all approaches (OC, SC and $\mathrm{AC}$ ).

An additional advantage of using a fictitious population is that, beside knowing the true population size, we can also calculate the TP estimator; the Petersen estimator with truly no linkage error. Since this is the maximum likelihood estimator using population 
information, the resulting estimate is the best estimate one could get. Thus, we use the TP estimator as a benchmark for the other estimators in our simulations. The TP estimator is based on the counts in Table 1 and does not equal the Petersen estimator one would get in practice using the counts from Table 2 .

\subsection{Results}

We performed 100 replications of the procedure mentioned in the previous subsection and, as expected, we indeed found that all 'optimal' parameters led to the same estimates in all four methods. In Table 4 the mean, median and standard deviation over the 100 replications is given for the difference between the estimates of the population size and the actual population size $N_{\mathcal{X}}=10,000$, for the estimators TP (method A, the benchmark), $\mathrm{P}$ (Petersen, using the counts from the linkage process), EM (method B), model (method C) and sample (method D). Note that TP and P are both based on Petersen's formula (Petersen 1896), but TP is using the (in practice unobservable) true population counts, whereas $\mathrm{P}$ uses the observed counts.

The first thing to notice is that the Petersen estimator using the observed counts indeed can lead to a heavily biased estimate of the population size, due to the linkage errors that are present. Moreover, we see that the EM-based estimator (method B) has a very large variance compared to the other estimators and at the same time has a larger bias. This indicates that this method is not well suited to be used for correcting linkage error.

Varying the relative size of the second register (i.e., the $f_{2}$ ) does not really influence the correction for linkage error. Indeed, the bias, as well as the variance of those estimators, seems to be more or less the same in all situations.

Table 4. Mean, median and variance of the difference with $N_{\mathcal{X}}=10,000$ of each estimator over the 100 replications, for different values of $f_{1}$ and $f_{2}$, and sample size 200.

\begin{tabular}{|c|c|c|c|c|c|c|c|}
\hline$f_{1}$ & $f_{2}$ & & $\mathrm{TP}-N_{\mathcal{X}}$ & $\mathrm{P}-N_{\mathcal{X}}$ & $\begin{array}{c}\text { EM } \\
-N_{\mathcal{X}}\end{array}$ & $\begin{array}{l}\text { model } \\
-N_{\mathcal{X}}\end{array}$ & $\begin{array}{l}\text { sample } \\
-N_{\mathcal{X}}\end{array}$ \\
\hline \multirow[t]{9}{*}{1.00} & \multirow[t]{3}{*}{0.90} & mean & 8.0 & 1399.0 & -1730.2 & 18.7 & 30.0 \\
\hline & & median & 9.2 & 1400.2 & -1812.5 & 23.0 & 13.4 \\
\hline & & st. dev. & 10.5 & 38.1 & 773.9 & 261.6 & 224.9 \\
\hline & \multirow[t]{3}{*}{0.50} & mean & 10.6 & 1193.1 & -1810.0 & 19.1 & -5.2 \\
\hline & & median & 12.6 & 1187.3 & -1891.6 & 33.8 & -28.0 \\
\hline & & st. dev. & 32.0 & 66.1 & 773.9 & 243.8 & 205.3 \\
\hline & \multirow[t]{3}{*}{0.15} & mean & 22.6 & 1053.5 & -2039.9 & 22.8 & 37.5 \\
\hline & & median & 17.2 & 1051.8 & -2109.6 & -1.1 & 31.8 \\
\hline & & st. dev. & 75.9 & 125.3 & 1326.7 & 259.1 & 198.2 \\
\hline \multirow[t]{3}{*}{0.50} & \multirow[t]{3}{*}{0.15} & mean & -17.8 & -129.5 & -1984.0 & -51.4 & 37.3 \\
\hline & & median & -28.5 & -147.5 & -2015.3 & -150.4 & -69.0 \\
\hline & & st. dev. & 269.1 & 258.6 & 1609.9 & 611.4 & 763.9 \\
\hline \multirow[t]{3}{*}{0.15} & \multirow[t]{3}{*}{0.15} & mean & 66.1 & -861.3 & -2638.6 & 212.5 & 450.3 \\
\hline & & median & 48.4 & -869.5 & -2683.5 & 183.7 & 299.3 \\
\hline & & st. dev. & 624.3 & 546.4 & 2116.4 & 1177.4 & 1709.6 \\
\hline
\end{tabular}




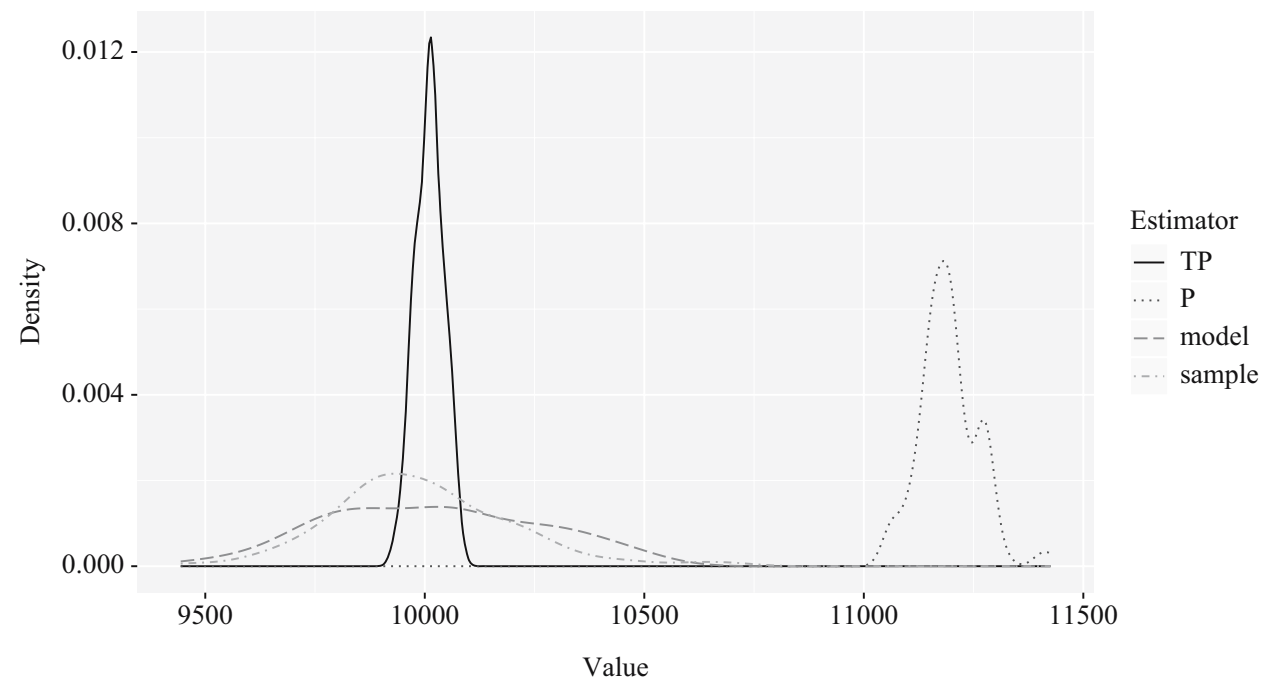

Fig. 1. Distributions of the P, TP, model based and sample based estimators for $N_{\mathcal{X}}=10,000, f_{1}=1.0$, $f_{2}=0.5$ and sample size 200 .

In case the registers include a unique identifier for some of the records, the identifier could be used as an alternative for taking a sample, under the assumption that the absence of the identifier is not (too) selective. When such a unique identifier is not present, it could, in practice, be quite costly to determine the true match status of pairs. Hence, probably only a small sample would be considered by a National Statistical Institute and that is why we used a relatively small sample from the second register for methods C and D.

Figure 1 shows a smooth estimate of the distribution of the estimators for $f_{1}=1.0$ and $f_{2}=0.5$. For the other values of $f_{2}$ the distributions look similar. We did not plot the EMbased estimator in this figure to be able to see more clearly the differences between the other estimation methods.

The figure again shows clearly that the Petersen estimator using the counts from the linkage process has a large bias (due to linkage error) and that the model and sample estimators nicely correct for that. The TP estimates are obviously performing the best, since they use the true knowledge about the number of matches. However, in practice that estimator is not possible.

Finally, we observe that in case both registers have low coverage $\left(f_{1}=f_{2}=0.15\right.$, that is, both registers contain less than 1400 records), the variance of the estimators that correct for linkage error increases considerably. This may not be that surprising when using such relatively small registers. However, the estimates that are supposed to correct for linkage error are still closer to the TP estimate than the Petersen estimate. Hence, we conclude that the correction methods work in all cases, albeit that they work best where at least one of the registers has high coverage.

\section{Conclusions}

In estimating the population size using capture-recapture, linkage errors (false links and missed links) affect the Petersen estimator. Indeed, the Petersen estimator then becomes 
heavily biased. To reduce that bias, some correction methods have been proposed in the literature. These methods introduce some additional parameters that should reflect the probability of occurrence of the two possible types of linkage error. They then model how linkage errors occur and use those error-probabilities to incorporate that model into the estimator. In the current article, we introduced a general formulation for such a correction method. That general formulation incorporates all previously introduced correction methods of that type as special cases.

Looking more closely to the general correction method, it turned out that the parameters could actually be chosen in such a way that the general estimator equals the optimal estimator, that is, the Petersen estimator with a known number of true matches. These optimal parameters can be estimated using different methods. We have shown that for at least two methods, the results improve the traditional Petersen estimator considerably. Those two methods make use of a relatively small sample for which the true match status of the records needs to be determined. More refined methods might even improve more and lead to estimators with smaller variances.

We have shown that it is possible to chose optimal parameters, such that all adjustment methods lead to exactly the same estimates. This reduces the need for making a choice on the error linkage model. However, in case the probabilities are estimated in a different way (e.g., by means of expert opinions), the different linkage error models will lead to different estimates. We have not investigated this further in the current article.

In case it is not possible to make use of a sample to estimate the optimal parameters, the general correction method could still be useful. In that situation, the model for the occurrence of the linkage errors should be assessed to estimate the error probabilities. We would like to note that the model assumes that double errors occur with negligible probabilities. With double errors we mean errors like missing a true match of a record and at the same time linking that record incorrectly to some record in the other register. In estimating the error-probabilities this should be taken into account in some way, because in practice such double errors do occur and would influence the error probabilities.

Using covariates or linking more than two registers would lead to more elaborate methods to estimate the population size in the presence of undercoverage. In these cases, more complex loglinear or Poisson models can be used to obtain a capture-recapture estimate. Similarly, the Fellegi and Sunter based linkage procedure can also be applied more elaborately, for example, by making use of blocking(s). This would affect the (estimates of the) posterior $m$-probabilities. In our view, the ideas expressed in the current article, as well as the introduced general formulation of the linkage error correction methods, will lead to a better understanding of the implications of such extensions and will be of help in deriving new, linkage error correcting, consistent estimators of the population size.

\section{Appendix}

\subsection{Appendix 1: Sets Defined in the Setting of Probabilistic Record Linkage}

Let $R_{1}$ be a register with records numbered $\{1,2,3, \ldots, 10\}$ and $R_{2}$ a register with records numbered $\{1,2,3, \ldots, 15\}$. The total number of pairs $(a, b)$ that can be constructed from the records of those registers is $10 \times 15=150$. Figure 2 shows all possible pairs. 


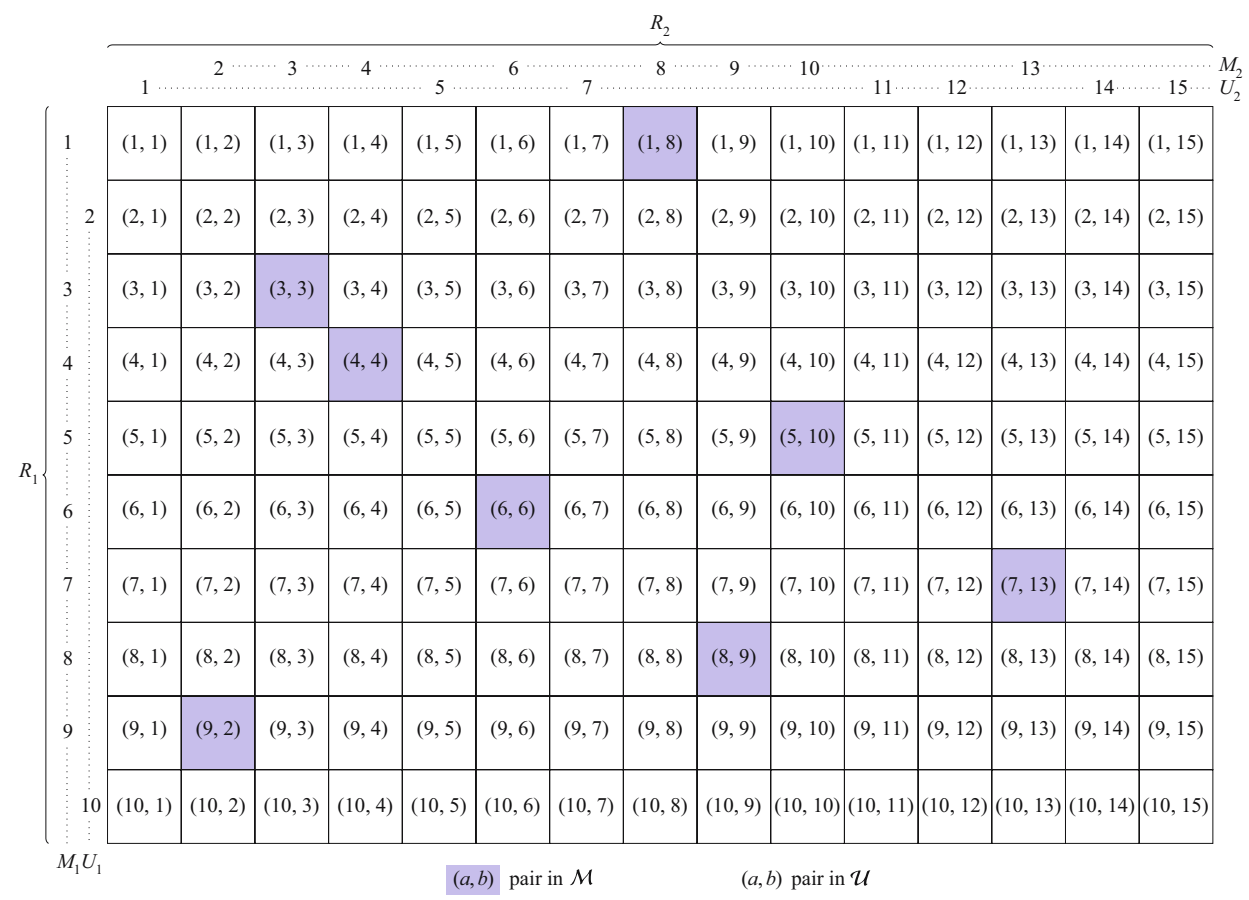

Fig. 2. Graphical representation of the sets of pairs defined in Subsection 2.2.

Moreover, an example of the set $\mathcal{M}$ of pairs of matching records and the set $\mathcal{U}$ of pairs of non-matching records is shown in that figure. In the example, the number of pairs in $\mathcal{M}$ is 8 and the number of pairs in $\mathcal{U}$ is 142 .

We can write each register as the union of two disjoint sets, $R_{i}=M_{i} \cup U_{i}$, where the disjoint sets of unique records are given by

$$
\begin{array}{ll}
M_{1}=\{1,3,4,5,6,7,8,9\} & U_{1}=\{2,10\} \\
M_{2}=\{2,3,4,6,8,9,10,13\} & U_{2}=\{1,5,7,11,12,14,15\}
\end{array}
$$

\subsection{Appendix 2: Admissibility of Asymmetric Two-way Correction Estimators $\hat{p}_{i}$}

The estimators for the probabilities $p_{i}$ in case of the asymmetric two-way correction approach should obviously be within $[0,1]$. This puts some restrictions on the parameters $\alpha, \beta_{1}$ and $\beta_{2}$.

To ensure that the estimators are non-negative, straightforward calculations lead to the condition that either

or

$$
\beta_{1} n_{1+}+\beta_{2} n_{+1} \leq n_{11} \quad \text { and } \quad \beta_{1}+\beta_{2}<\alpha
$$

$$
\beta_{1} n_{1+}+\beta_{2} n_{+1} \geq n_{11} \text { and } \quad \beta_{1}+\beta_{2}>\alpha
$$

Additionally, ensuring that both probabilities are not larger than one, leads under (22) to the condition

$$
\beta_{1} n_{1+}+\beta_{2} n_{+1} \geq n_{11}-\left(\alpha-\left(\beta_{1}+\beta_{2}\right)\right)\left(n_{1+} \wedge n_{+1}\right)
$$


and under (23) to the condition

$$
\beta_{1} n_{1+}+\beta_{2} n_{+1} \leq n_{11}-\left(\alpha-\left(\beta_{1}+\beta_{2}\right)\right)\left(n_{1+} \vee n_{+1}\right)
$$

where $n_{1+} \vee n_{+1}$ equals the maximum of $n_{1+}$ and $n_{+1}$ and $n_{1+} \wedge n_{+1}$ the minimum of $n_{1+}$ and $n_{+1}$.

Summarizing, we need either

$$
\left.\begin{array}{l}
\beta_{1}+\beta_{2}<\alpha \\
\beta_{1} n_{1+}+\beta_{2} n_{+1} \leq n_{11} \\
\beta_{1} n_{1+}+\beta_{2} n_{+1} \geq n_{11}-\left(\alpha-\left(\beta_{1}+\beta_{2}\right)\right)\left(n_{1+} \wedge n_{+1}\right)
\end{array}\right\}
$$

or

$$
\left.\begin{array}{l}
\beta_{1}+\beta_{2}>\alpha \\
\beta_{1} n_{1+}+\beta_{2} n_{+1} \geq n_{11} \\
\beta_{1} n_{1+}+\beta_{2} n_{+1} \leq n_{11}-\left(\alpha-\left(\beta_{1}+\beta_{2}\right)\right)\left(n_{1+} \vee n_{+1}\right)
\end{array}\right\}
$$

Assuming $R_{1}$ to be the largest data set, that is, $n_{1+}>n_{+1}$, the set of conditions (26) is equivalent to

$$
\left.\begin{array}{l}
\beta_{1} \geq\left(n_{11}-\alpha n_{+1}\right) /\left(n_{1+}-n_{+1}\right) \\
\beta_{1}+\beta_{2}<\alpha \\
\beta_{1} n_{1+}+\beta_{2} n_{+1} \leq n_{11}
\end{array}\right\}
$$

and the set of conditions (27) to

$$
\left.\begin{array}{l}
\beta_{2} \geq\left(\alpha n_{1+}-n_{11}\right) /\left(n_{1+}-n_{+1}\right) \\
\beta_{1}+\beta_{2}>\alpha \\
\beta_{1} n_{1+}+\beta_{2} n_{+1} \geq n_{11}
\end{array}\right\}
$$

Assuming the two data sets to be of equal size, that is, $n_{1+}=n_{+1}$, the set of conditions (26) is equivalent to

$$
\left.\begin{array}{l}
\alpha \geq n_{11} / n_{1+} \\
\beta_{1}+\beta_{2}<\alpha \\
\beta_{1} n_{1+}+\beta_{2} n_{+1} \leq n_{11}
\end{array}\right\}
$$

and the set of conditions (27) to

$$
\left.\begin{array}{l}
\alpha \leq n_{11} / n_{1+} \\
\beta_{1}+\beta_{2}<\alpha \\
\beta_{1} n_{1+}+\beta_{2} n_{+1} \geq n_{11}
\end{array}\right\}
$$




\subsection{Appendix 3: Enforcing One-To-One Linkage}

In our asymmetric two-way correction method, we have three parameters: $\alpha, \beta_{1}$ and $\beta_{2}$. In case we enforce one-to-one linkage, we can actually do with two, because in that situation we can write $\beta_{1}$ as a function of $\alpha$ and $\beta_{2}$.

In Figure 3 the relation between (expected) counts based on the population and based on linkage are shown in the situation where we potentially would like to apply the asymmetric two-way correction with enforced one-to-one linkage. Under the assumption of one-to-one linkage, it should hold that $\beta_{1} N_{10}=\beta_{2} N_{01}$, as can be seen in the figure. Noting that $\mathbb{E} N_{10}=p_{1}\left(1-p_{2}\right) N_{\mathcal{X}}$ and $\mathbb{E} N_{01}=p_{2}\left(1-p_{1}\right) N_{\mathcal{X}}$ and plugging in the estimators $\hat{p}_{1}$ and $\hat{p}_{2}$ from (17), we can derive the following relation

$$
\beta_{1}=\frac{\left(\alpha n_{+1}-n_{11}\right) \beta_{2}}{\left(\alpha n_{1+}-n_{11}\right)-2 \beta_{2}\left(n_{1+}-n_{+1}\right)}
$$

Note that, assuming equal sizes of the two registers, i.e., $n_{1+}=n_{+1}$, Equation (28) yields $\beta_{1}=\beta_{2}$. That is, we would obtain the situation in which the symmetric two-way correction is applicable.

Moreover, from (28) it follows that

$$
\begin{aligned}
& \alpha>2 \beta_{2} \text { and } n_{1+}>n_{+1} \Rightarrow \beta_{1}<\beta_{2} \\
& \alpha>2 \beta_{2} \text { and } n_{1+}<n_{+1} \Rightarrow \beta_{1}>\beta_{2}
\end{aligned}
$$

as expected (see discussion in Section 1).

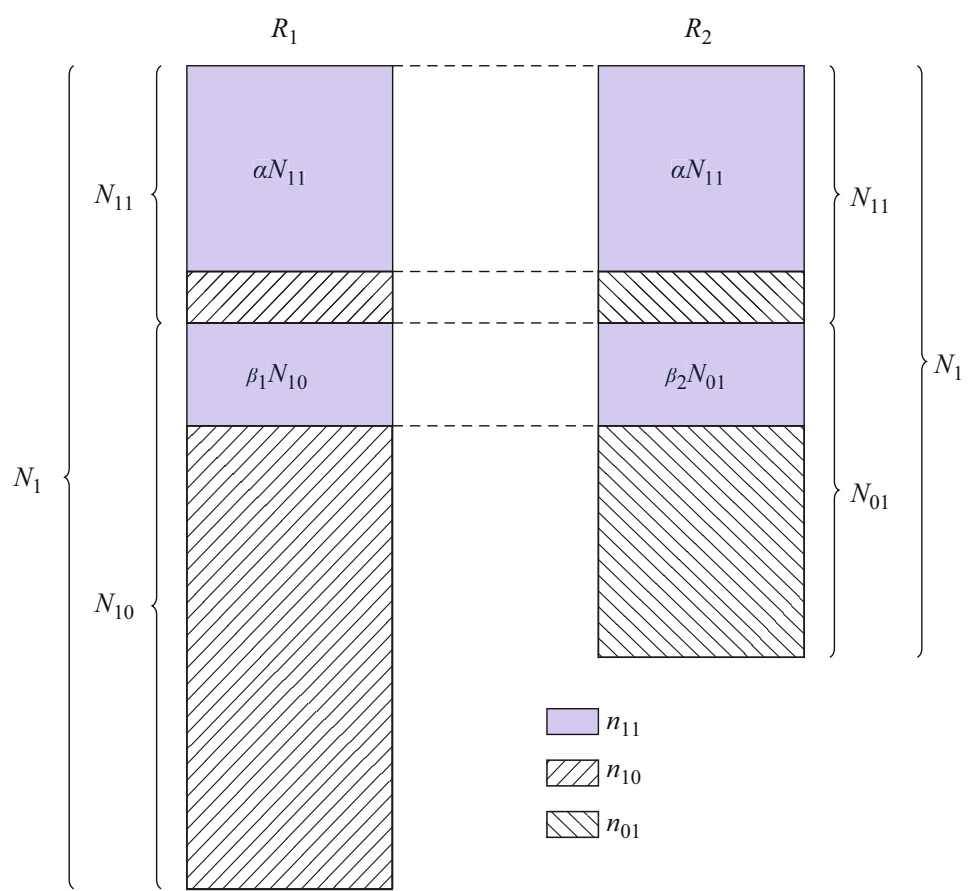

Fig. 3. Relations between counts based on population and based on one-to-one linkage. 


\subsection{Appendix 4: Estimation of the Matching Probabilities Using Logistic Regression}

For a sample of records from the smallest register it is assumed that the true match status can be determined, that is, we assume that it is known whether or not the record should be linked to a record from the larger register and if so, with which record it should be linked. Therefore, for a subset of all pairs generated in the linkage process, the true match status is known. The goal of the logistic regression model is to predict the probability that this pair is a true match, based on properties of the record pair.

In the regression model the following covariates are used

1. The result of the comparison of the linkage variables. In this case the linkage variables are the three elements of the date of birth; day (DB_D), month (DB_M) and year (DB_Y). These variables are binary; both records of the pair agree on the variables (true) or not (false). If in at least one of the records a variable is missing, we consider it a disagreement (false).

2. Whether or not the pair is selected when enforcing one-to-one linkage (LNK). This is also a binary variable which is false when there is a more likely match for one or both of the records. This variable is a strong predictor for true matches.

The target variable is the true match status (a binary variable). The model is estimated using the sampled pairs and then used to calculate predictions of the matching probability for all pairs. The main goal of the model is to correct for differences in the population between the sample and the complete set of pairs. This should result in a more accurate estimate of the number of linkage errors. As the model is merely used as an illustration of a method that can be used to estimate the relevant parameters for the correction methods, the model is kept relatively simple. Therefore, all variables are added as main effects and no interactions are used in the model.

To estimate $m_{11}$ the probability that a pair is a true match given that a pair has been linked is needed, and to estimate $N_{11}$ the probability of a true match given that a pair has been linked or has not been linked is needed. Therefore, as long as the sample is representative for the set of pairs, using only LNK should be enough to obtain unbiased estimates of $N_{11}$ and $m_{11}$ ( $n_{11}$ follows directly from the linkage procedure). Adding additional variables to the regression model, such as DB_D, DB_M and DB_Y in this case, could lead to a reduction of the variance of the estimators when the probability of a false link depends on this variable and when the sample is not representative with respect to these variables. Other variables (sex and age) were investigated, but as these did not affect the outcomes in this study, these are not included in the final results.

\section{References}

Cadwell, B.L., P.J. Smith, and A.L. Baughman. 2005. "Methods for Capture-Recapture Analysis When Cases Lack Personal Identifiers." Statistics in Medicine 24(13): 2041-2051. Doi: https://doi.org/10.1002/sim.2081.

Di Consiglio, L. and T. Tuoto. 2015. "Coverage Evaluation on Probabilistically Linked Data.” Journal of Official Statistics 31(3): 415-429. Doi: https://doi.org/10.1515/jos2015-0025. 
Ding, Y. and S.E. Fienberg. 1994. "Dual System Estimation of Census Undercount in the Presence of Matching Error." Survey Methodology 20: 149-158. Available at: https://www150.statcan.gc.ca/n1/en/pub/12-001-x/1994002/article/14422-eng.pdf (accessed June 2019).

Fienberg, S.E. 1992. "Bibliography on Capture-Recapture Modelling with Application to Census Undercount Adjustment." Survey Methodology 18: 143-154. Available at: https://www150.statcan.gc.ca/n1/pub/12-001-x/1992001/article/14494-eng.pdf (accessed June 2019).

Fellegi, I.P. and A.B. Sunter. 1969. “A Theory for Record Linkage.” Journal of the American Statistical Association 64: 1183-1210. Doi: https://doi.org/10.1080/ 01621459.1969.10501049.

Gerritse, S.C., B.F.M. Bakker, P.P. de Wolf, and P.G.M. van der Heijden. 2016a. "Under Coverage of the Population Register in the Netherlands, 2010." Discussion paper 2016-02 (Centraal Bureau voor de Statistiek, Den Haag/Heerlen). Available at: https://www.cbs.nl/nl-nl/achtergrond/2016/08/under-coverage-of-the-population-register-in-the-netherlands-2010 (accessed June 2019).

Gerritse, S.C., B.F.M. Bakker, D. Zult, and P.G.M. van der Heijden. 2016b. The Effects of Imperfect Linkage and Erroneous Captures on the Population Size Estimator, Chapter 3 of $\mathrm{PhD}$ thesis An Application of Population Size Estimation to Official Statistics, S.C. Gerritse, ISBN 978-94-6233-323-9. Available at: https://dspace.library.uu.nl/bitstream/handle/1874/337476/Gerritse.pdf (accessed June 2019).

Herzog, T.N., F.J. Scheuren, and W.E. Winkler. 2007. Data Quality and Record Linkage Techniques. Springer. Doi: https://doi.org/10.1007/0-387-69505-2.

Lincoln, F.C. 1930. "Calculating Waterfowl Abundance on the Basis of Banding Returns." United States Department of Agriculture Circular 118: 1-4. Available at: https://archive.org/details/calculatingwater118linc/page/n1.

McLeod, P., D. Heasman, and I. Forbes. 2011. Simulated Data for the on the Job Training, ESSnet DI. Available at: https://ec.europa.eu/eurostat/cros/content/job-training_en (accessed 15 April 2019).

Petersen, C.G. J. 1896. "The Yearly Immigration of Young Plaice into the Limfjord from the German Sea." Report of the Danish Biological Station (1895) 6: 5-84. Available at: https://www.biodiversitylibrary.org/ia/reportofdanishbi06dans\#page/8/mode/1 up (accessed June 2019).

Sanathanan, L. 1972. "Estimating the Size of a Multinomial Population." The Annals of Mathematical Statistics 43(1): 142-152. Doi: https://doi.org/10.1214/aoms/1177 692709.

Wolter, K.M. 1986. "Some Coverage Error Models for Census Data." Journal of the American Statistical Association 81: 338-346. Doi: https://doi.org/10.1080/01621459. 1986.10478277.

Received July 2018

Revised January 2019

Accepted April 2019 